\title{
Phytopathology
}

\section{Artificial Surfaces in Phyllosphere Microbiology}

\author{
Hung K. Doan and Johan H. J. Leveau
}

Department of Plant Pathology, University of California, Davis, CA 95616.

Accepted for publication 10 April 2015.

\begin{abstract}
Doan, H. K., and Leveau, J. H. J. 2015. Artificial surfaces in phyllosphere microbiology. Phytopathology 105:1036-1042.

The study of microorganisms that reside on plant leaf surfaces, or phyllosphere microbiology, greatly benefits from the availability of artificial surfaces that mimic in one or more ways the complexity of foliage as a microbial habitat. These leaf surface proxies range from very simple, such as nutrient agars that can reveal the metabolic versatility or antagonistic properties of leaf-associated microorganisms, to the very complex, such as silicon-based casts that replicate leaf surface topography down to nanometer resolution. In this review, we summarize the various uses of artificial surfaces in experimental phyllosphere microbiology and discuss how these have advanced our understanding of the biology of leaf-associated microorganisms and the habitat they live in. We also provide an outlook into future uses of artificial leaf surfaces, foretelling a greater role for microfluidics to introduce biological and chemical gradients into artificial leaf environments, stressing the importance of artificial surfaces to generate quantitative data that support computational models of microbial life on real leaves, and rethinking the leaf surface ('phyllosphere') as a habitat that features two intimately connected but very different compartments, i.e., the leaf surface landscape ('phylloplane') and the leaf surface waterscape ('phyllotelma').
\end{abstract}

Once considered a neglected milieu (Ruinen 1961), the phyllosphere, or leaf surface as a microbial habitat, has gained considerable popularity as a subject of scientific inquiry. In the past decades, the number of studies that cite research papers on the topics of phyllosphere and its (pseudo)synonym phylloplane has been growing exponentially, doubling in size roughly every 6 years. Several explanations for this dramatic increase may be considered. One is the long-overdue realization that the phyllosphere represents a model microbial biome that is highly amenable to experiment- and simulation-driven approaches to answer outstanding questions not just in plant microbiology, but in ecology in general (Meyer and Leveau 2012). A second explanation is the growing concern among not only scientists and funding agencies, but also health officials and consumers over the contamination of raw-consumed leafy greens with enteropathogenic bacteria (Heaton and Jones 2008) and the need to understand in mechanistic terms the attachment and survival of these unwanted contaminants on plant leaf surfaces. Last but not least, existing or newly emerging foliar diseases remain an enormous challenge for plant pathologists whose goal is to prevent foliar pathogens from diminishing health and yield of economically important crops and ecologically valued trees (Newton et al. 2010).

Corresponding author: J. H. J. Leveau; E-mail address: jleveau@ucdavis.edu

http://dx.doi.org/10.1094/PHYTO-02-15-0050-RVW

(C) 2015 The American Phytopathological Society
Several excellent reviews and book chapters have been published recently on phyllosphere microbiology (Heaton and Jones 2008; Leveau 2015; Meyer and Leveau 2012; Muller and Ruppel 2014; Rastogi et al. 2013; Vorholt 2012; Whipps et al. 2008). A brief summary of the current state of knowledge is that the phyllosphere is a very complex environment that requires unique adaptations for microbial survival and that is characterized by extreme and erratic variation both spatially and temporally in the conditions that microbes (e.g., bacteria, fungi, and yeast) might encounter during their residence on a leaf and that impact their experience, fate, and interactions with each other and their host.

To deconstruct the complexity and interplay of physical, chemical, and biological processes that occur on leaves, many researchers have resorted to proxy surfaces that are simpler and more defined than real leaves. Such artificial surfaces allow a reductionist approach toward identifying the impacts of individual factors (be they physical, chemical, or biological) on the success or failure of microorganisms to attach to, survive, and reproduce on leaf surfaces. Artificial surfaces may be as simple as solidified agar or inert glass or as complicated as plastic replicas of a leaf surface which are microscopically nearly indistinguishable from real leaves.

In this review, we provide a synopsis and some key examples of the various uses of artificial surfaces in phyllosphere microbiology and how they have improved our understanding of microbial life on plant foliage. We will limit our review to that part of the scientific literature which deals with artificial leaf surfaces that are inoculated and colonized by actual microorganisms. This excludes from our 
review an in-depth analysis of the simulated or in silico leaf environment (Box 1).

\section{SIMPLE SURFACES}

The first type of artificial surface that we will discuss is one that we refer to as "simple" and which we define as completely lacking lateral heterogeneity at the time of inoculation. In other words, these surfaces are flat (minimal topography), their chemical landscape is uniform, and they are sterile (not previously colonized by microorganisms). It is important to note that our definition includes the descriptor 'at the time of inoculation', because inoculation of even these simple surfaces inevitably introduces complexity and heterogeneity: for example, inoculants will settle in some but not other spots on the surface, locally consume nutrients and produce secondary metabolites, and generate topography by formation of colonies (biofilms) or manipulation of the surface.

Nutrient agar. Invented in the late 1800 s by coworkers in the laboratory of Robert Koch (Hitchens and Leikind 1939), solidified nutrient agar represents one of the most important and simplest of surfaces to be used as a means to study phyllosphere microorganisms. As early as 1904 (Duggeli 1904), microbiologists were rinsing plant leaves in water or buffered solutions and spreadplating the leaf washes onto nutrient agar to discover large and diverse populations of colony-forming microorganisms that naturally associate with plant foliage. Numbers and species of microorganisms that appeared on the agar surfaces varied with the amounts and types of nutrients that were included in the agar, which demonstrated the richness and variation in metabolic versatility among leaf-associated microorganisms (Ercolani 1991). Also, the prevalence of colored colonies among leaf isolates (Goodfellow et al. 1976) exposed the importance of pigmentation as a phyllosphere-related adaptation against harmful ultraviolet (UV) radiation from sunlight (Jacobs et al. 2005; Moody et al. 1999). As an alternative to leaf washing, leaves were also pressed directly onto nutrient agar surfaces (Corpe 1985), or first contact-printed onto balloons or velvet cloth (Rusch and Leben 1968). These experiments offered early insights into the nonrandom distribution of microorganisms on leaf surfaces. For example, microbial colonization was heavier near veins or on the abaxial (bottom) side of leaves (Leben 1988).

Nutrient agar has also proven useful as a substratum-based arena to investigate the interactions between leaf-associated microorganisms. The driving force behind these types of studies often is the desire to identify and characterize biocontrol agents of foliar diseases. Typically, these experiments consist of pairwise confrontations between colonies of plant pathogens and presumed antagonists or their culture filtrates or volatiles (Pandey et al. 1993). Colony interactions have been described as ranging from no mutual impact on growth to inhibition at a distance (Skidmore and Dickinson 1976). Importantly, interactions observed on agar are not always accurate predictors of how two microorganisms may interact on leaves (Pandey et al. 1993). While this observation underscores the limitations of nutrient agar as a proxy for real leaves, it also provokes the following question: what is it about real leaves (complex topography? native microbial communities? plant-specific exudates?) that would explain the different outcomes of microbial interactions on agar versus leaf surfaces?

One phyllosphere-specific modification of nutrient agars that deserves special mention is the substitution of agar with molten paraffin to create a surface that aims to resemble the waxy quality of actual leaves (McCormack et al. 1994). This artificial substrate was used successfully and in a variety of ways for the in vitro study of microbial activities and interactions in the phyllosphere. For example, on this substrate, supplemented with nutrients to support growth, leaf-isolated yeasts clearly outcompeted yeasts isolated from soil, which suggests that the former were better adapted to the waxy and hydrophobic nature of the paraffin-based surface (McCormack et al. 1994).
Nutrient agar also was used in a series of experiments that were designed to address lateral dispersion of bacterial cells on leaf surfaces, specifically by comparing it to bacterial behavior on an agar surface. Inoculation of leaves with a mixture of green and red fluorescent bacteria resulted in patterns of colonization that showed clusters made up of one or two colors, whereas only single-colored (red or green) colonies were observed on agar (Tecon and Leveau 2012). Also, use of a green fluorescent protein (GFP)-based bacterial bioreporter of reproductive success (Remus-Emsermann and Leveau 2010; Remus-Emsermann et al. 2012) revealed that the GFP content in individual cells on agar surfaces was inversely proportional to the size of the cluster that those cells were in. In contrast, that correlation was lost on leaves, and many cells were less fluorescent than would be expected based on the size of the cluster to which the cells belonged (Tecon and Leveau 2012). Both experiments demonstrated the ability of bacteria to relocate on leaves, presumably by exploitation of the waterscape as a matrix for dispersion.

In the era of "omics"-based profiling of microbial communities, nutrient agar plates serve an important function for estimating the fraction of microorganisms in a particular environment that are not readily culturable. For bacteria on lettuce leaves, that fraction has been estimated to be between 90 and 99.9\% (Rastogi et al. 2010). Among the "nonculturable" microbes are representatives from taxa that cannot be grown in the absence of their host, for example, obligate parasites from the fungal order Erysiphales, or from taxa that require nutrients other than those provided in the agar, e.g., alkane-degrading bacteria from the genus Alkanindiges. Nutrient agars also have served as control surfaces in studies that profile the transcriptome or proteome of microorganisms as they colonize the leaf surface (Gourion et al. 2006; Scheublin et al. 2014).

Inert surfaces made of metal, plastic, glass, and other materials. In contrast to nutrient agar, which is designed to provide microorganisms with one or more sources of carbon, nitrogen and energy for growth, inert surfaces, as defined here, share the characteristic that they merely represent an opportunity for attachment. If microbial growth is important for the experiment that uses these types of surfaces, nutrients may be provided externally, for example in the same suspension in which the microorganisms are deposited onto the surface. Inert surfaces can be made out of a variety of materials, including metal, glass, and plastic, and treated to change surface properties such as hydrophobicity to assess the impact of

\section{BOX 1}

Computational simulations in support of phyllosphere microbiology are relatively few. PHYLLOSIM (van der Wal et al. 2013) is a Netlogobased model of a leaf surface landscape that features spatial and temporal variation in the availability of water and nutrients and that is colonized by bacteria that use the water landscape for lateral dispersion and leaf-leached nutrients for growth. A major insight obtained through PHYLLOSIM has been the notion that in order to explain experimental observations of bacterial cluster sizes on plant leaves, one must assume that with some frequency individual bacteria detach from growing cell clusters and relocate to other sites on the same leaf to start a new cluster. A similar prediction was derived independently from another leaf surface model (PerezVelazquez et al. 2012). Although phyllosphere-specific models are relatively rare, there is no shortage of models that simulate microbial colonization of surfaces (Klausen et al. 2006; Kreft et al. 1998), including surfaces that feature lateral variation in physical, chemical and biological constraints (Wang and Or 2013). Hopefully, it will be only a matter of time before these models will be adopted, modified, and used along existing phyllosphere models such as PHYLLOSIM, to make bottom-up predictions and answer open questions about microbial behaviors on plant leaves. 
such modifications on attachment or growth of microorganisms. For this section, we only consider surfaces that are flat, i.e., without significant topographical heterogeneity.

One major application for inert surfaces is in the study of microorganisms' ability to attach to surfaces and/or to create surfacebased biofilms (Morris and Monier 2003). Much of the most recent phyllosphere literature on this topic features microbial contaminants of leafy greens such as human pathogenic Escherichia coli and Salmonella, with the primary goal to identify intrinsic or extrinsic factors that contribute to attachment and, equally important, resistance to detachment. For example, stainless steel was used to demonstrate variation among strains of $E$. coli in surface attachment (Rivas et al. 2007), and to show that fibrous cell surface proteins known as curli were not involved in this attachment (Ryu et al. 2004). Another example is the use of polystyrene surfaces of microtiter plates to quantify biofilm formation, for example in the study of the interaction of Salmonella with cut Romaine lettuce (Kroupitski et al. 2009). For E. coli, it was demonstrated that the set of genes that is important for binding to these types of plastic surfaces overlaps with, but is not identical to, the set of genes that is important in the same bacterium for binding to plant tissue (Torres et al. 2005). This finding is significant and warns against a direct translation of data from artificial surfaces into theories about microbial life on real leaves.

Several studies go beyond the question of leaf surface attachment and ask how microorganisms detach from surfaces. A recent study (Brandl and Huynh 2014) quantified the impact of Tween 80 on biofilms of Salmonella enterica on cilantro leaves and showed that use of this surfactant promoted the dislodging of bacterial cells from biofilm aggregates. In another study, GFP-based bioreporter technology was used to demonstrate the attachment and detachment by phyllosphere bacteria of Pantoea agglomerans to a solid agarose surface (van der Wal et al. 2013). Agarose is a purified form of agar and can be considered an inert surface as defined here, and nutrients were provided in the same drop of water that also harbored the bacteria. Importantly, this study provided evidence that bacteria were reproducing in the drop, i.e., the waterscape of the leaf, rather than on the leaf surface itself. This has important implications for how we think about life on leaf surfaces. Many of our models emphasize the aspect of surface-based growth and activity, while many processes might actually play out in the waterscape.

Not all studies that use inert and flat surfaces deal with microbial attachment. Glass slides were inoculated with water or bacterial suspensions in water to show that leaf-isolated microorganisms can lower the contact angle of liquid drops (Knoll and Schreiber 1998). This was explained in the context of the phenomenon of leaf wetting (Taylor 2011) and how microorganisms may contribute to this process. Also, the experiment exposed these types of bacteria as "ecosystem engineers" (Jones et al. 1994), able to modify their local environment supposedly to their benefit. Lowering the contact angle of a drop of water increases the surface area covered by that drop, which in simulation models has been shown to increase the amount of nutrients that leach from the interior of the leaf to the surface where they become available to surface colonizers (van der Wal and Leveau 2011). Glass surfaces also play a supporting role in other technologies that have been developed in recent years to study phyllosphere microorganisms. For example, fluid force microscopy (FluidFM) is a method for the isolation of individual bacteria from among a mixture of leaf-associated microorganisms that are deposited in a drop of leaf wash on a microscope slide (Stiefel et al. 2013). Interestingly, it was shown using FluidFM that twothirds of the single bacteria in leaf washes were able to grow in R2A liquid medium compared with a mere $10 \%$ recovery rate from dilution plating of the same samples on R2A plates. This finding clearly challenges the typically low estimates of culturability based on agar plate counts. The ability to pick out single cells from among a large and diverse microbial community holds enormous promise for the future characterization of difficult-to-culture microorganisms that reside on leaf surfaces.
Several studies involving inert surfaces describe the interactions of phyllosphere-isolated microorganisms not with the surface but with each other. For example, coinoculation of glass slides with bacteria and ${ }^{14} \mathrm{C}$-labeled fungal spores revealed that ${ }^{14} \mathrm{C}$ leaked from the spores and was utilized by and incorporated into the bacterial biomass (Brodie and Blakeman 1975). This is an elegant demonstration of a trophic interaction that would be difficult to demonstrate on real leaf surfaces. In a more recent study (Carter et al. 2012), stainless steel slides (also called coupons) were used as a proxy surface to reveal that the presence of a normal spinach microbiota stimulated the attachment of $E$. coli but negatively impacted biofilm formation by $E$. coli. The source of nutrients in this experiment was spinach leaf lysate that was filtered (or not) to remove (or not) the microbial communities that naturally associate with spinach leaves. Interestingly, the presence of $E$. coli also appeared to have a negative impact on the diversity of the microbial community (Carter et al. 2012). Furthermore, many of the genes that were upregulated in the E. coli-invaded spinach microbiota appeared to be involved in the metabolism of spinach nutrients. Both findings suggest that the pathogen and indigenous spinach microbes competed for the same limited resources.

A newer type of artificial surface is porous ceramic, which is designed to represent a surface that can be readily modulated to study microbial processes under variable water potential (Dechesne et al. 2008). Even in the absence of obvious wetness, a leaf may be patchily covered with thin water films that might result from interactions between salt crystals on the leaf surface and water vapor from the atmosphere and the stomata (Burkhardt et al. 1999). These types of differently saturated conditions make life on the leaf surface challenging for microorganisms. In a modified version of the porous surface setup, one that featured an agar slab on top of the ceramic plate (Dechesne and Smets 2012), the ability of the model phyllosphere bacterium Pseudomonas syringae B728a to swarm was severely impacted at matric potentials lower than $-0.1 \mathrm{kPa}$, which corresponds to a relative humidity of less than $99.99992 \%$. As swarming motility is thought to be important for this bacterium to explore leaf surfaces (Hockett et al. 2013), the results from these experiments suggest that bacterial relocation on the leaf depends heavily on the availability of sites with very high ambient humidity.

\section{MICROFABRICATED SURFACES}

We consider microfabricated surfaces to be any substratum that is created or recreated from synthetic or plant material to feature lateral variability in one or more physical, chemical, or biological condition. These surfaces are exploited primarily to reveal how spatial and temporal heterogeneity impacts microbial growth, survival, and interactions. Most of the examples that we will discuss represent surfaces that vary laterally in micro- or nanometer topography, including reconstituted leaf waxes, isolated leaf cuticles, and lithography-generated landscapes with patterned structures representing mimics of stomata, trichomes, and grooves. However, we also consider microfluidic devices, despite the fact that these are not yet widely used in phyllosphere microbiology.

Reconstituted wax surfaces. Epicuticular waxes can be extracted from plant leaves and recrystallized on glass (Blakeman and Sztejnberg 1973). These reconstituted wax surfaces closely resemble those of intact leaves in terms of the crystalline patterns at nanometer resolution, yet they lack major structures such as stomata, trichomes and veins (Jeffree 1974). Because of this reduction in complexity, artificial wax surfaces could be important tools for the study of interactions between microorganisms and epicuticular waxes. For example, they may be used to validate experimental differences between wild-type and wax mutants of maize in the ability to allow bacterial establishment and maintenance on their leaves (Beattie 2002). Reconstituted wax also has been used to assess the surface behavior of rust fungi (Reisige et al. 2006). In this elegant study, it was shown that 
epicuticular waxes from wheat leaves induced appressoria formation and infection hyphae in Puccinia graminis f. sp. tritici. No such induction was observed on dental wax, and comparison of the two wax types revealed the $\mathrm{C} 28$ aldehyde octacosanal as a plant-specific wax component responsible for the difference in fungal behavior. In another study (Blakeman and Sztejnberg 1973), it was suggested that leaf waxes may have fungistatic activity, i.e., they negatively impacted spore germination by Botrytis cinerea, and this inhibition could be overcome by the addition of nutrients. In the same study, spore germination was shown to be greater on real leaves than on reconstituted wax surfaces, suggesting an additional source of antifungal activity on the real leaves, most probably bacteria with fungistatic properties.

Leaf peels and isolated leaf cuticles. Several protocols exist for the isolation of leaf epidermis and cuticles (Hauser et al. 1993; Remus-Emsermann et al. 2011; Weyers and Travis 1981). Leaf peels consist of epidermal strips that have been used in phyllosphere microbiology to observe and quantify the response of functional stomata to the presence and activity of bacteria and to demonstrate the importance of stomata as ports of bacterial entry into the leaf interior (Melotto et al. 2006). Isolated leaf cuticles have been used to quantitatively determine the rates by which water and sugars diffuse from the leaf interior to the surface and become available for microorganisms. Typically, the setup for these cuticle experiments involves two compartments that are called source and sink and are separated by the cuticle (Remus-Emsermann et al. 2011; van der Wal and Leveau 2011). Not all plant species can be used for these studies; their cuticles may be too thin to handle or both sides of their leaves feature stomata that would produce holes in the isolated cuticle. In one setup (Schreiber et al. 2005), diffusion of water across cuticles of Hedera and Prunus was shown to greatly increase when leaf cuticles were inoculated with bacteria. The production of cuticle-degrading enzymes or biosurfactants has been proposed as a possible mechanism for this bacteria-dependent increase in cuticle permeability. Isolated cuticles from walnut (Juglans regia) were used to estimate the permeability of the leaf sugar fructose as $10^{-6} \mathrm{~m} / \mathrm{h}$ (van der Wal and Leveau 2011). Estimates like these are very few but crucial for computational models (Box 1) that simulate sugar diffusion to the leaf surface to show that even at such low diffusion rates, sufficient amounts of sugars make it to the leaf surface to sustain microbial population sizes as they are typically found in the phyllosphere (van der Wal and Leveau 2011). Another study (Remus-Emsermann et al. 2011) estimated the permeability of fructose across isolated cuticles of poplar (Populus $\times$ canescens), but also employed bacterial bioreporters to demonstrate that diffusion of the sugar did not occur homogeneously across the leaf cuticle but instead was limited to certain sites, most notably the base of trichomes (Remus-Emsermann et al. 2011). Assuming that these sites were the only ones leaking sugars, it was deduced that values for permeability could at least locally be as high as $3 \times 10^{-3} \mathrm{~m} / \mathrm{h}$. This notion of extreme heterogeneous availability of nutrients is consistent with the observed variability in reproductive success (RemusEmsermann and Leveau 2010; Remus-Emsermann et al. 2012) and access to nutrients (Leveau and Lindow 2001) of bacterial colonizers of the leaf surface.

Photolithography. Photolithography is a multistep process for the microfabrication of geometrical shapes on the surface of an inert material. Often this material is silicon, which is first covered with a layer of light-sensitive material called photoresist, then exposed to ultraviolet light through a mask that covers some but not all areas of the surface. The UV-exposed portions of the surface are then etched away or treated to allow deposition of a new material. Either way, the result is a three-dimensional landscape of structures with varying widths, heights and patterns, based on the design of the mask that was used. This microlandscaped wafer can be used as-is (Sirinutsomboon et al. 2011) or as a negative master mold into or over which materials such as dental wax or polydimethylsiloxane (PDMS) are pressed or poured to produce a positive replica of the landscape (Weibel et al. 2007). While photolithography is routinely used in the fabrication of microdevices to answer (micro)biological questions, only few examples exist of its use specifically in the study of leaf surface colonization by microorganisms. One is from the field of food safety, where E. coli was inoculated onto miniature landscapes of silicon that featured regularly patterned rudimentary trichomes, stomata, or grooves (Sirinutsomboon et al. 2011). These experiments showed that $E$. coli attached better to surfaces that featured stomata or grooves than to surfaces with trichomes. However, on surfaces with trichomes or stomata, cells appeared to be preferentially clustered around the base of trichomes and in a zone of 5 to 10 micrometer distance from stomatal openings; no such attraction was seen with grooves (Sirinutsomboon et al. 2011). In another study with E. coli (Komaromy et al. 2009), bacteria attached better to surfaces with densely patterned pyramid-shaped structures than to less densely patterned or flat surfaces. Both studies highlight the impact of surface structures on the colonization patterns of microorganisms. They also imply that when we observe colonization patterns on real leaves, we should consider the possibility that these resulted from purely physical interactions between the bacteria, the surface and the waterscape.

Many studies have looked at the interaction of fungi with glass or plastic surfaces to assess the impact of topography on fungal behavior. For example, appressorium formation varied among several species of rust fungi in response to surface ridges with defined heights (Allen et al. 1991). The findings were consistent with the idea that some fungi rely much more than others on leaf surface topography (compared with chemotropic signals) and that a wider thigmotropic sensitivity (i.e., responsiveness to a wide range of leaf topographies) may be beneficial in fungal adaptation to a wider plant host range (Allen et al. 1991). In an experiment that saw the plant pathogenic foliar fungus Colletotrichum graminicola inoculated onto a silicon landscape with micropillars of different heights and widths, the induction of appressoria by the fungus correlated strongly with pillar width, such that it took 4 micrometers of continuous contact area to form these appressoria (Apoga et al. 2004). This insight is now an important part of the mechanistic model for appressorium formation in foliar pathogenic fungi (Tucker and Talbot 2001).

Microfluidics. Microfluidics refers to a technology that allows control of the flow of fluids along micrometer dimensions with the goal to mimic naturally water-covered microbial habitats. This control can introduce biological and chemical variation in time and space and allow the study of its impact on the habitat's microorganisms in real-time (Rusconi et al. 2014). Microfluidics has not yet made it into the standard tool box of phyllosphere microbiologists, but there are many ways in which to exploit this technology to advance our understanding of leaf surface colonization. Of special interest is the use of microfluidics in combination with microfabricated surfaces, and one example is a study (Scheuerman et al. 1998) that looked at the impact of grooves of different widths (10 to $40 \mu \mathrm{m})$ and a fixed depth $(10 \mu \mathrm{m})$ on the surface colonization by Pseudomonas species in a flow that was perpendicular to the direction of the grooves. Only motile Pseudomonas cells (never nonmotile Pseudomonas cells) were found at the bottom of the grooves, indicating that colonization of certain features of the surface waterscape requires an active role on the part of microorganisms. Another exciting example of microfluidics as it could be applied to phyllosphere microbiology is the fabrication of a compartmentalized miniature landscape where many microhabitats are connected by small corridors that allow bacterial movement between microhabitats, each of which is differentially supplied with nutrients through so-called nanoslits so as to create a nutritionally heterogeneous environment (Keymer et al. 2006). In such an environment, two competing $E$. coli strains, one of which would always outcompete the other in a liquid culture, actually segregated spatially and were able to coexist (Keymer et al. 2008). If we were to extrapolate this finding to the phyllosphere, we might hypothesize that the heterogeneity of the leaf surface, in terms of 
where and how much nutrients are available, is a key factor explaining the microbial diversity that is associated with plant foliage (Delmotte et al. 2009; Hunter et al. 2010; Kembel and Mueller 2014; Lambais et al. 2014; Rastogi et al. 2012; Redford et al. 2010; Williams and Marco 2014).

\section{REPLICA SURFACES}

The topography of plant leaves is extremely complex (Koch et al. 2008a) and very difficult if not impossible to reproduce de novo using a technique like photolithography. However, the use of leaf surfaces as templates in casting protocols has been very successful. Such casting involves the use of a conditionally soft and deformable material, for example dental wax (Koch et al. 2007; Koch et al. 2008b) or PDMS (McDonald et al. 2013) that is pressed into or poured over the surface of the template leaf. This gives a negative imprint that now can serve in a second casting process as template to create a positive replica of the leaf, out of PDMS or another material. Initial interest in these techniques sprung from the desire to create a superhydrophobic surface with self-cleaning and antimicrobial properties, similar to that of lotus leaves (Sun et al. 2005). Artificial leaves produced by this replica casting technique show the leaf surface and all of its microand nanostructures in exquisite detail (Schulte et al. 2009). Only recently have groups started to inoculate these artificial leaf surfaces with microorganisms to assess the differences and similarities in surface colonization compared with real leaves. For example, PDMS casts of spinach leaves were inoculated with $E$. coli to reveal that bacteria tended to concentrate in the grooves between plant epidermal cells (Zhang et al. 2014). In the same study, negative leaf casts of spinach leaves were used to prepare positive nutrient agar surfaces with exact leaf topography. On these artificial surfaces, the increase in bacterial population sizes was similar to that on flat agar surfaces, but on the artificial surfaces, growth was concentrated in the areas that corresponded with grooves. Bacteria that were buried deeply inside these grooves were better protected from treatment with a biocide than cells that were growing on a flat agar surface.

\section{CONCLUSION AND OUTLOOK}

We hope to have made a convincing case for the usefulness of artificial surfaces toward a fuller understanding of the phyllosphere as a microbial habitat. Substrata as simple as nutrient agars or as complex as PDMS-casted leaves can be exploited in numerous ways to observe and compare the abundances, (inter)activities, and distributions of microorganisms on real versus artificial leaves. From the differences and similarities between the two, we can deduce working principles of leaf surface colonization by microorganisms. We anticipate that the field of phyllosphere microbiology will embrace and even combine some of the newer types of artificial surfaces and microfluidics, eventually venturing into experimental setups that go beyond the inoculation of one or two microbial strains, but instead introduce synthetic communities (Bodenhausen et al. 2014) or even native communities directly from leaf washes (Williams and Marco 2014). The use of PDMS-cast leaves is highly compatible with phyllosphere-relevant techniques such as GFPbased bioreporters (Leveau and Lindow 2001) and fluorescence in situ hybridization (Remus-Emsermann et al. 2014), which holds great promise for the generation of quantitative data that can be used to validate simulation models for phyllosphere microbiology.

One of the challenges for future generations of artificial leaf surfaces is to achieve greater control and understanding of what we have been referring to in this review as the leaf surface waterscape. Water is necessary for life in the phyllosphere, and it plays an important role in the ability of microorganisms to relocate, access nutrients and sense or communicate with other microorganisms (Beattie 2011). In this context, the term 'phylloplane', which is oftentimes used synonymously with phyllosphere, is probably best used to describe the actual physical boundary of the leaf, i.e., the cuticle, to which microorganisms may attach in some demonstrable way. 'Phyllosphere' microorganisms could then include all the cuticle-attached microbes in addition to those that are present in the waterscape. This idea of two distinct but interconnected compartments within the phyllosphere is not new. Some species of carnivorous and other plants feature modified leaves that form pitchers to trap a body of water that serves as a habitat for microorganisms and other microfauna (Gray et al. 2012). The highly salinous drops of dew that form on leaves of the Tamarix tree have been referred to as 'miniature lakes' (Qvit-Raz et al. 2008). A separate term, referring to the collective body of water that is attached to and influenced by a leaf surface and in which phyllosphere microorganisms may reside planktonically, would appear to be useful, and so the term 'phyllotelma' is proposed here, from the Greek words 'phyllo' (meaning leaf) and 'telma' (meaning 'pond' or 'standing water'; also see the definition of 'phytotelmata' by Maguire [1971], as 'plant-held waters', i.e., small bodies of water held by leaves or flowers of plants and host to a variety of aquatic organisms). The demonstration that leaf bacteria can readily detach from surfaces and continue life in water drops on the surface (van der Wal et al. 2013) begs for a closer look at the phylloplane and phyllotelma as separate but intimately connected compartments of the phyllosphere. Experimentally, this will be a challenging task but one that will be greatly facilitated by the availability of artificial leaf surfaces.

\section{ACKNOWLEDGMENTS}

We acknowledge funding from USDA-NIFA award number 2014-67017-21695.

\section{LITERATURE CITED}

Allen, E. A., Hazen, B. E., Hoch, H. C., Kwon, Y., Leinhos, G. M. E., Staples, R. C., Stumpf, M. A., and Terhune, B. T. 1991. Appressorium formation in response to topographical signals by 27 rust species. Phytopathology 81 : 323-331.

Apoga, D., Barnard, J., Craighead, H. G., and Hoch, H. C. 2004. Quantification of substratum contact required for initiation of Colletotrichum graminicola appressoria. Fungal Genet. Biol. 41:1-12.

Beattie, G. A. 2002. Leaf surface waxes and the process of leaf colonization by microorganisms. Pages 3-26 in: Phyllosphere Microbiology. S. E. Lindow, E. I. Hecht-Poinar, and V. J. Elliott, eds. American Phytopathological Society, St. Paul, MN.

Beattie, G. A. 2011. Water relations in the interaction of foliar bacterial pathogens with plants. Annu. Rev. Phytopathol. 49:533-555.

Blakeman, J. P., and Sztejnberg, A. 1973. Effect of surface wax on inhibition of germination of Botrytis cinerea spores on beetroot leaves. Physiol. Plant Pathol. 3:269-278.

Bodenhausen, N., Bortfeld-Miller, M., Ackermann, M., and Vorholt, J. A. 2014. A synthetic community approach reveals plant genotypes affecting the phyllosphere microbiota. PLoS Genet. 10:e1004283.

Brandl, M. T., and Huynh, S. 2014. Effect of the surfactant Tween 80 on the detachment and dispersal of Salmonella enterica serovar Thompson single cells and aggregates from cilantro leaves as revealed by image analysis. Appl. Environ. Microbiol. 80:5037-5042.

Brodie, I. D. S., and Blakeman, J. P. 1975. Competition for carbon compounds by a leaf surface bacterium and conidia of Botrytis cinerea. Physiol. Plant Pathol. 6:125-135.

Burkhardt, J., Kaiser, H., Goldbach, H., and Kappen, L. 1999. Measurements of electrical leaf surface conductance reveal recondensation of transpired water vapour on leaf surfaces. Plant Cell Environ. 22:189-196.

Carter, M. Q., Xue, K., Brandl, M. T., Liu, F. F., Wu, L. Y., Louie, J. W., Mandrell, R. E., and Zhou, J. Z. 2012. Functional metagenomics of Escherichia coli O157:H7 interactions with spinach indigenous microorganisms during biofilm formation. PLoS One 7:e44186.

Corpe, W. A. 1985. A method for detecting methylotrophic bacteria on solid surfaces. J. Microbiol. Methods 3:215-221.

Dechesne, A., Or, D., Gulez, G., and Smets, B. F. 2008. The porous surface model, a novel experimental system for online quantitative observation of microbial processes under unsaturated conditions. Appl. Environ. Microbiol. 74:5195-5200. 
Dechesne, A., and Smets, B. F. 2012. Pseudomonad swarming motility is restricted to a narrow range of high matric water potentials. Appl. Environ. Microbiol. 78:2936-2940.

Delmotte, N., Knief, C., Chaffron, S., Innerebner, G., Roschitzki, B., Schlapbach, R., von Mering, C., and Vorholt, J. A. 2009. Community proteogenomics reveals insights into the physiology of phyllosphere bacteria. Proc. Natl. Acad. Sci. USA 106:16428-16433.

Duggeli, M. 1904. Die Bakterienflora gesunder Samen und daraus gezogener Keimplanzchen. Zentralblatt fur Bakteriologie u. Par. II. Abt. Bd. 12u. 13.

Ercolani, G. L. 1991. Distribution of epiphytic bacteria on olive leaves and the influence of leaf age and sampling time. Microbiol. Ecol. 21:35-48.

Goodfellow, M., Austin, B., and Dickinson, C. H. 1976. Numerical taxonomy of some yellow-pigmented bacteria isolated from plants. J. Gen. Microbiol. 97:219-233.

Gourion, B., Rossignol, M., and Vorholt, J. A. 2006. A proteomic study of Methylobacterium extorquens reveals a response regulator essential for epiphytic growth. Proc. Natl. Acad. Sci. USA 103:13186-13191.

Gray, S. M., Akob, D. M., Green, S. J., and Kostka, J. E. 2012. The bacterial composition within the Sarracenia purpurea model system: Local scale differences and the relationship with the other members of the food web. PLoS One 7:e50969.

Hauser, H. D., Walters, K. D., and Berg, V. S. 1993. Patterns of effective permeability of leaf cuticles to acids. Plant Physiol. 101:251-257.

Heaton, J. C., and Jones, K. 2008. Microbial contamination of fruit and vegetables and the behaviour of enteropathogens in the phyllosphere: A review. J. Appl. Microbiol. 104:613-626.

Hitchens, A. P., and Leikind, M. C. 1939. The introduction of agar-agar into bacteriology. J. Bacteriol. 37:485-493.

Hockett, K. L., Burch, A. Y., and Lindow, S. E. 2013. Thermo-regulation of genes mediating motility and plant interactions in Pseudomonas syringae. PLoS One 8:e59850.

Hunter, P. J., Hand, P., Pink, D., Whipps, J. M., and Bending, G. D. 2010. Both leaf properties and microbe-microbe interactions influence within-species variation in bacterial population diversity and structure in the lettuce (Lactuca species) phyllosphere. Appl. Environ. Microbiol. 76:8117-8125.

Jacobs, J. L., Carroll, T. L., and Sundin, G. W. 2005. The role of pigmentation, UV radiation tolerance, and leaf colonization strategies in the epiphytic survival of phyllosphere bacteria. Microbiol. Ecol. 49:104-113.

Jeffree, C. E. 1974. Method for recrystallising selected components of plant epicuticular waxes as surfaces for the growth of micro-organisms. Trans. Br. Mycol. Soc. 63:626-629.

Jones, C. G., Lawton, J. H., and Shachak, M. 1994. Organisms as ecosystem engineers. Oikos 69:373-386.

Kembel, S. W., and Mueller, R. C. 2014. Plant traits and taxonomy drive host associations in tropical phyllosphere fungal communities. Botany 92: 303-311.

Keymer, J. E., Galajda, P., Lambert, G., Liao, D., and Austin, R. H. 2008. Computation of mutual fitness by competing bacteria. Proc. Natl. Acad. Sci. USA 105:20269-20273.

Keymer, J. E., Galajda, P., Muldoon, C., Park, S., and Austin, R. H. 2006. Bacterial metapopulations in nanofabricated landscapes. Proc. Natl. Acad. Sci. USA 103:17290-17295.

Klausen, M., Gjermansen, M., Kreft, J. U., and Tolker-Nielsen, T. 2006. Dynamics of development and dispersal in sessile microbial communities: Examples from Pseudomonas aeruginosa and Pseudomonas putida model biofilms. FEMS Microbiol. Lett. 261:1-11.

Knoll, D., and Schreiber, L. 1998. Influence of epiphytic micro-organisms on leaf wettability: Wetting of the upper leaf surface of Juglans regia and of model surfaces in relation to colonization by micro-organisms. New Phytol. 140:271-282.

Koch, K., Bhushan, B., and Barthlott, W. 2008a. Diversity of structure, morphology and wetting of plant surfaces. Soft Matter 4:1943-1963.

Koch, K., Dommisse, A., Barthlott, W., and Gorb, S. 2007. The use of plant waxes as templates for micro- and nanopatterning of surfaces. Acta Biomater. 3:905-909.

Koch, K., Schulte, A. J., Fischer, A., Gorb, S. N., and Barthlott, W. 2008b. A fast, precise and low-cost replication technique for nano- and high-aspectratio structures of biological and artificial surfaces. Bioinspir. Biomim. 3: 046002 .

Komaromy, A., Boysen, R. I., Zhang, H., McKinnon, I., Fulga, F., Hearn, M. T. W., and Nicolau, D. V. 2009. Micro-structures modulate bacterial cell viability and attachment. Microelectron. Eng. 86:1431-1434.

Kreft, J. U., Booth, G., and Wimpenny, J. W. T. 1998. BacSim, a simulator for individual-based modelling of bacterial colony growth. Microbiol. UK 144: 3275-3287.

Kroupitski, Y., Pinto, R., Brandl, M. T., Belausov, E., and Sela, S. 2009. Interactions of Salmonella enterica with lettuce leaves. J. Appl. Microbiol. 106:1876-1885
Lambais, M. R., Lucheta, A. R., and Crowley, D. E. 2014. Bacterial community assemblages associated with the phyllosphere, dermosphere, and rhizosphere of tree species of the Atlantic forest are host taxon dependent. Microbiol. Ecol. 68:567-574.

Leben, C. 1988. Relative humidity and the survival of epiphytic bacteria with buds and leaves of cucumber plants. Phytopathology 78:179-185.

Leveau, J., and Lindow, S. 2001. Appetite of an epiphyte: Quantitative monitoring of bacterial sugar consumption in the phyllosphere. Proc. Natl. Acad. Sci. USA 98:3446-3453.

Leveau, J. H. J. 2015. Life of microbes on aerial plant parts. Pages 17-24 in: Principles of Plant-Microbe Interactions-Microbes for Sustainable Agriculture. Lugtenberg, ed. Springer, Cham, Switzerland.

Maguire, B. 1971. Phytotelmata: Biota and community structure determination in plant-held waters. Annu. Rev. Ecol. Syst. 2:439-464.

McCormack, P. J., Bailey, M. J., and Jeffries, P. 1994. An artificial wax substrate for the in vitro study of phylloplane micro-organisms. J. Microbiol. Methods 19:19-28.

McDonald, B., Patel, P., and Zhao, B. 2013. Micro-structured polymer film mimicking the trembling aspen leaf. Chem. Eng. Process Tech. 1:1012.

Melotto, M., Underwood, W., Koczan, J., Nomura, K., and He, S. Y. 2006. Plant stomata function in innate immunity against bacterial invasion. Cell 126:969-980.

Meyer, K. M., and Leveau, J. H. J. 2012. Microbiology of the phyllosphere: A playground for testing ecological concepts. Oecologia 168:621-629.

Moody, S. A., Newsham, K. K., Ayres, P. G., and Paul, N. D. 1999. Variation in the responses of litter and phylloplane fungi to UV-B radiation (290315 nm). Mycol. Res. 103:1469-1477.

Morris, C. E., and Monier, J.-M. 2003. The ecological significance of biofilm formation by plant-associated bacteria. Annu. Rev. Phytopathol. 41: 429-453.

Muller, T., and Ruppel, S. 2014. Progress in cultivation-independent phyllosphere microbiology. FEMS Microbiol. Ecol. 87:2-17.

Newton, A. C., Gravouil, C., and Fountaine, J. M. 2010. Managing the ecology of foliar pathogens: Ecological tolerance in crops. Ann. Appl. Biol. 157: 343-359.

Pandey, R. R., Arora, D. K., and Dubey, R. C. 1993. Antagonistic interaction between fungal pathogens and phyloplane fungi of guava. Mycopathologia 124:31-39.

Perez-Velazquez, J., Schlicht, R., Dulla, G., Hense, B. A., Kuttler, C., and Lindow, S. E. 2012. Stochastic modeling of Pseudomonas syringae growth in the phyllosphere. Math. Biosci. 239:106-116.

Qvit-Raz, N., Jurkevitch, E., and Belkin, S. 2008. Drop-size soda lakes: Transient microbial habitats on a salt-secreting desert tree. Genetics 178: 1615-1622.

Rastogi, G., Coaker, G. L., and Leveau, J. H. J. 2013. New insights into the structure and function of phyllosphere microbiota through high-throughput molecular approaches. FEMS Microbiol. Lett. 348:1-10.

Rastogi, G., Sbodio, A., Tech, J. J., Suslow, T. V., Coaker, G. L., and Leveau, J. H. 2012. Leaf microbiota in an agroecosystem: Spatiotemporal variation in bacterial community composition on field-grown lettuce. ISME J. 6: 1812-1822.

Rastogi, G., Tech, J. J., Coaker, G. L., and Leveau, J. H. 2010. A PCR-based toolbox for the culture-independent quantification of total bacterial abundances in plant environments. J. Microbiol. Methods 83:127-132.

Redford, A. J., Bowers, R. M., Knight, R., Linhart, Y., and Fierer, N. 2010. The ecology of the phyllosphere: Geographic and phylogenetic variability in the distribution of bacteria on tree leaves. Environ. Microbiol. 12:2885-2893.

Reisige, K., Gorzelanny, C., Daniels, U., and Moerschbacher, B. M. 2006. The C28 aldehyde octacosanal is a morphogenetically active component involved in host plant recognition and infection structure differentiation in the wheat stem rust fungus. Physiol. Mol. Plant Pathol. 68:33-40.

Remus-Emsermann, M. N., and Leveau, J. H. 2010. Linking environmental heterogeneity and reproductive success at single-cell resolution. ISME J. 4: 215-222.

Remus-Emsermann, M. N., Tecon, R., Kowalchuk, G. A., and Leveau, J. H. 2012. Variation in local carrying capacity and the individual fate of bacterial colonizers in the phyllosphere. ISME J. 6:756-765.

Remus-Emsermann, M. N. P., de Oliveira, S., Schreiber, L., and Leveau, J. H. J. 2011. Quantification of lateral heterogeneity in carbohydrate permeability of isolated plant leaf cuticles. Front. Microbiol. 2:197.

Remus-Emsermann, M. N. P., Lucker, S., Muller, D. B., Potthoff, E., Daims, H., and Vorholt, J. A. 2014. Spatial distribution analyses of natural phyllosphere-colonizing bacteria on Arabidopsis thaliana revealed by fluorescence in situ hybridization. Environ. Microbiol. 16:2329-2340.

Rivas, L., Fegan, N., and Dykes, G. A. 2007. Attachment of Shiga toxigenic Escherichia coli to stainless steel. Int. J. Food Microbiol. 115:89-94.

Ruinen, J. 1961. The phyllosphere. I. An ecologically neglected milieu. Plant Soil 15:81-109. 
Rusch, V., and Leben, C. 1968. Epiphytic microflora: The balloon print isolation technique. Can. J. Microbiol. 14:486-487.

Rusconi, R., Garren, M., and Stocker, R. 2014. Microfluidics expanding the frontiers of microbial ecology. Annu. Rev. Biophys. 43:65-91.

Ryu, J. H., Kim, H., Frank, J. F., and Beuchat, L. R. 2004. Attachment and biofilm formation on stainless steel by Escherichia coli $\mathrm{O} 157: \mathrm{H} 7$ as affected by curli production. Lett. Appl. Microbiol. 39:359-362.

Scheublin, T. R., Deusch, S., Moreno-Forero, S. K., Muller, J. A., van der Meer, J. R., and Leveau, J. H. J. 2014. Transcriptional profiling of Gram-positive Arthrobacter in the phyllosphere: Induction of pollutant degradation genes by natural plant phenolic compounds. Environ. Microbiol. 16:2212-2225.

Scheuerman, T. R., Camper, A. K., and Hamilton, M. A. 1998. Effects of substratum topography on bacterial adhesion. J. Colloid Interface Sci. 208: 23-33.

Schreiber, L., Krimm, U., Knoll, D., Sayed, M., Auling, G., and Kroppenstedt, R. M. 2005. Plant-microbe interactions: Identification of epiphytic bacteria and their ability to alter leaf surface permeability. New Phytol. 166: 589-594.

Schulte, A. J., Koch, K., Spaeth, M., and Barthlott, W. 2009. Biomimetic replicas: Transfer of complex architectures with different optical properties from plant surfaces onto technical materials. Acta Biomater. 5: 1848-1854.

Sirinutsomboon, B., Delwiche, M. J., and Young, G. M. 2011. Attachment of Escherichia coli on plant surface structures built by microfabrication. Biosys. Eng. 108:244-252.

Skidmore, A. M., and Dickinson, C. H. 1976. Colony interactions and hyphal interference between Septoria nodorum and phylloplane fungi. Trans. Br. Mycol. Soc. 66:57-64.

Stiefel, P., Zambelli, T., and Vorholt, J. A. 2013. Isolation of optically targeted single bacteria by application of fluidic force microscopy to aerobic anoxygenic phototrophs from the phyllosphere. Appl. Environ. Microbiol. 79:4895-4905.

Sun, M., Luo, C., Xu, L., Ji, H., Ouyang, Q., Yu, D., and Chen, Y. 2005. Artificial lotus leaf by nanocasting. Langmuir 21:8978-8981.
Taylor, P. 2011. The wetting of leaf surfaces. Curr. Opin. Colloid In. 16: 326-334.

Tecon, R., and Leveau, J. H. J. 2012. The mechanics of bacterial cluster formation on plant leaf surfaces as revealed by bioreporter technology. Environ. Microbiol. 14:1325-1332.

Torres, A. G., Jeter, C., Langley, W., and Matthysse, A. G. 2005. Differential binding of Escherichia coli O157:H7 to alfalfa, human epithelial cells, and plastic is mediated by a variety of surface structures. Appl. Environ. Microbiol. 71:8008-8015.

Tucker, S. L., and Talbot, N. J. 2001. Surface attachment and pre-penetration stage development by plant-pathogenic fungi. Annu. Rev. Phytopathol. 39:385-417.

van der Wal, A., and Leveau, J. H. J. 2011. Modelling sugar diffusion across plant leaf cuticles: The effect of free water on substrate availability to phyllosphere bacteria. Environ. Microbiol. 13:792-797.

van der Wal, A., Tecon, R., Kreft, J. U., Mooij, W. M., and Leveau, J. H. J. 2013. Explaining bacterial dispersion on leaf surfaces with an individualbased model (PHYLLOSIM). PLoS One 8:e75633.

Vorholt, J. A. 2012. Microbial life in the phyllosphere. Nat. Rev. Microbiol. 10:828-840.

Wang, G., and Or, D. 2013. Hydration dynamics promote bacterial coexistence on rough surfaces. ISME J. 7:395-404.

Weibel, D. B., DiLuzio, W. R., and Whitesides, G. M. 2007. Microfabrication meets microbiology. Nat. Rev. Microbiol. 5:209-218.

Weyers, J. D. B., and Travis, A. J. 1981. Selection and preparation of leaf epidermis for experiments in stomatal physiology. J. Exp. Bot. 32:837-850.

Whipps, J. M., Hand, P., Pink, D., and Bending, G. D. 2008. Phyllosphere microbiology with special reference to diversity and plant genotype. J. Appl. Microbiol. 105:1744-1755.

Williams, T. R., and Marco, M. L. 2014. Phyllosphere microbiota composition and microbial community transplantation on lettuce plants grown indoors. MBio 5:e01564-14.

Zhang, B., Luo, Y., Pearlstein, A. J., Aplin, J., Liu, Y., Bauchan, G. R., Payne, G. F., Wang, Q., Nou, X., and Millner, P. D. 2014. Fabrication of biomimetically patterned surfaces and their application to probing plantbacteria interactions. ACS Appl. Mater. Interfaces 6:12467-12478. 